\title{
Steady-State Plasma Concentrations of Polyethylene Glycol (PEG) are Reached in Children and Adults During Once-Weekly Prophylactic Treatment with Nonacog Beta Pegol (N9-GP)
}

\author{
Ola Sternebring ${ }^{1}\left[\right.$ Charlotte Gabel-Jensen ${ }^{1} \cdot$ Helene Jacobsen ${ }^{1} \cdot$ Andrew James Benie $^{1} \cdot$ Inga Bjørnsdottir $^{1}$
}

Published online: 23 September 2019

(c) The Author(s) 2019

\begin{abstract}
Background Nonacog beta pegol (N9-GP, Refixia ${ }^{\circledR}$, Rebinyn ${ }^{\circledR}$ ) is a human recombinant coagulation factor IX (rFIX) conjugated to a 40-kDa polyethylene glycol (PEG) moiety. PEGylation significantly prolongs the circulation half-life compared with conventional FIX replacement treatments, resulting in higher FIX levels. Although there is extensive clinical experience with PEGylated molecules, the potential for abnormal and/or indefinite PEG accumulation during long-term treatment and the hypothetical impact on long-term safety is still under discussion.

Aim The aim of this study was to examine plasma PEG concentrations in children, adolescents and adults undergoing onceweekly intravenous prophylactic treatment with N9-GP for up to 6.5 years.

Methods Plasma samples were collected as part of the PARADIGM clinical development programme (PARADIGM 2/4 [NCT01333111 and NCT01395810] and PARADIGM 5 [NCT01467427]). Proton nuclear magnetic resonance ( $\left.{ }^{1} \mathrm{H}-\mathrm{NMR}\right)$ was used to measure plasma PEG concentrations.

Results Steady-state plasma PEG concentrations were reached approximately 6 months after initiation of weekly prophylactic treatment with 40 IU/kg N9-GP. Mean steady-state plasma PEG concentrations were $5.6 \mu \mathrm{g} / \mathrm{mL}$ in children $\leq 12$ years old at enrolment (PARADIGM 5) and $5.3 \mu \mathrm{g} / \mathrm{mL}$ in adolescents/adults $>12$ years old (PARADIGM 2/4). Plasma PEG concentrations tended to be lower in younger children $<7$ years old (mean $4.6 \mu \mathrm{g} / \mathrm{mL}$ ). There was a correlation between plasma PEG and FIX activity levels in all age groups.

Conclusion PEG steady-state plasma levels were maintained for up to 6.5 years during continuous prophylactic treatment and PEG levels correlated with FIX activity. Apart from the initial increase to steady state, no further systemic PEG accumulation was observed.
\end{abstract}

\section{Introduction}

Haemophilia B is a rare, recessive bleeding disorder characterised by a deficiency in functional coagulation factor IX (FIX). Patients with severe haemophilia B exhibit FIX clotting activity at $<1 \%$ of normal levels [1]. Nonacog beta pegol (N9-GP; Refixia ${ }^{\circledR}$; Rebinyn ${ }^{\circledR}$ ) is a recombinant coagulation FIX (rFIX) with a branched 40-kDa polyethylene glycol (PEG) moiety that is conjugated to the activation peptide via site-directed glycoPEGylation. During the coagulation process, the activation peptide and the attached PEG molecule are released from N9-GP, leaving native activated FIX.

Inga Bjørnsdottir

INBJ@novonordisk.com

1 Novo Nordisk A/S, Novo Nordisk Park, 2760 Måløv, Denmark
PEGylation of rFIX significantly prolongs the circulation half-life in humans approximately fivefold compared with standard rFIX, maintaining elevated FIX activity for longer $[2,3]$. This allows for less frequent dosing compared with conventional rFIX or plasma-derived FIX (pdFIX) products $[2,4,5]$. Weekly prophylaxis with N9-GP 40 IU/kg maintains trough FIX activity levels beyond the current treatment goal ( $>1 \%$ of normal levels) to more recently suggested goals of $12-15 \%$ and above [6]. Phase III clinical trials have confirmed the efficacy, tolerability and safety of N9-GP for the prevention and on-demand treatment of bleeding episodes in paediatric, adolescent and adult patients with haemophilia B [2, $7,8]$. Patients on weekly prophylaxis with N9-GP $40 \mathrm{IU} / \mathrm{kg}$ achieved mean FIX activity trough levels of $17 \%$ in children and $27 \%$ in adolescents and adults, resulting in low annualised bleeding rates and resolution of target joints $[2,7,9]$. 


\section{Key Points}

Plasma polyethylene glycol (PEG) concentrations were examined in children, adolescents and adults undergoing once-weekly intravenous prophylactic treatment with nonacog beta pegol (N9-GP) (Refixia ${ }^{\circledR}$, Rebinyn ${ }^{\circledR}$ ), a PEGylated coagulation factor IX product.

PEG levels reached steady state in plasma around 6 months after N9-GP treatment initiation and no further accumulation was detected during continuous prophylactic treatment lasting up to 6.5 years.

Plasma PEG concentrations were shown to correlate with factor IX activity levels in patients of all ages.

PEGylation remains the most commonly used method with which to extend the half-life of biopharmaceuticals [10]. Several PEGylated drugs have received approval from the European Medicines Agency (EMA) and/or the US Food and Drug Administration (FDA) since the early 1990s [11-14]. Despite extensive clinical experience with marketed PEGylated products in adults [15, 16], some authorities have highlighted that PEG could potentially accumulate beyond expected steady-state levels in plasma and tissues during long-term treatment, raising hypothetical concerns regarding safety $[14,16]$. However, it is important to note here that no safety concerns attributable to PEG accumulation have been reported for any marketed PEGylated drug.

To date, there are very few patient data published that describe the relationship between chronic administration of PEGylated peptide/protein products and PEG steady-state levels in plasma and tissues. Steady-state PEG levels are expected when the input rate (dose and dose frequency) is in equilibrium with the output rate (elimination rate from tissues and plasma) $[15,16]$. While the time to reach steady state is generally governed by the terminal half-life of the compound, steady-state levels are determined by dose, dose frequency and clearance of the drug.

With respect to N9-GP, evidence from a non-clinical animal model suggests that PEG levels reach a steady state in plasma and tissues during chronic treatment. Single-dose distribution and excretion studies in N9-GP-treated rats showed that 40-kDa PEG was widely distributed, cleared from plasma and tissues over time and excreted in urine and faeces [17]. The fact that PEG is removed from plasma and tissues further indicates that steady-state levels would be expected after repeated dosing. Because an assessment of PEG tissue levels in clinical trials is not feasible, plasma measurements may be used as a surrogate marker for tissue exposure, with the assumption that PEG elimination pathways in humans and rats are similar.

Here, we present PEG plasma exposure data from patients treated prophylactically with once-weekly intravenous administration of N9-GP for a period of up to 6.5 years. The main aim of this analysis was to examine whether plasma 40-kDa PEG concentrations in patients receiving N9-GP reach steady-state levels. A secondary objective was to assess the relationship between the PEG levels and FIX activity levels in plasma. Proton nuclear magnetic resonance $\left({ }^{1} \mathrm{H}-\mathrm{NMR}\right)$, a method that has been previously shown to reliably measure total PEG (conjugated and free) in plasma [18, 19], was used to analyse samples collected as part of the Novo Nordisk PARADIGM clinical development programme.

\section{Materials and Methods}

\subsection{Trial Design, Patients and Sample Collection}

PARADIGM 2 (ClinicalTrials.gov identifier: NCT01333111) [2], PARADIGM 4 (NCT01395810) [9] and PARADIGM 5 (NCT01467427) [7] were multinational, open-label clinical trials designed to investigate the safety, efficacy and pharmacokinetics of N9-GP in paediatric, adolescent and adult male patients with haemophilia B. PARADIGM 5 (paediatric patients $\leq 12$ years old at enrolment; FIX activity $\leq 2 \% ; N=25)$ comprised a main phase of 52 weeks, followed by an ongoing extension phase. Patients were treated prophylactically with N9-GP $40 \mathrm{IU} / \mathrm{kg}$ once weekly. Bleeding episodes were treated with a single $40-\mathrm{IU} / \mathrm{kg}$ N9-GP dose or with $80 \mathrm{IU} / \mathrm{kg}$ for a severe bleeding episode. Although some patients became adolescents over the course of PARADIGM 5, we refer to this study as the paediatric trial throughout the manuscript. In the PARADIGM 2 trial (patients $>12-70$ years old; FIX activity $\leq 2 \% ; N=74$ ), the patient and treating physician chose an on-demand or prophylactic treatment regimen. Patients selecting prophylactic treatment were randomised $1: 1$ to receive 10 or $40 \mathrm{IU} / \mathrm{kg}$ once-weekly N9-GP over 52 weeks. The duration of ondemand treatment was 28 weeks. Only patients receiving weekly prophylactic N9-GP $40 \mathrm{IU} / \mathrm{kg}$ were included in the analysis presented here. Bleeding episodes were treated with a single N9-GP dose of 40 or $80 \mathrm{IU} / \mathrm{kg}$, depending on severity. PARADIGM 4 was an extension of PARADIGM 2 [9].

According to the PARADIGM 5 trial protocol, biospecimen samples could be used for exploratory studies. Informed consent was obtained for analysis of samples collected for antibody analysis. As part of the PARADIGM 5 trial, pre-dose (trough) plasma samples were collected from 19 
paediatric patients (aged 1-11 years old at trial initiation) over a period of up to 6.5 years starting from trial initiation. Approval from ethical committees was obtained for analysis of plasma samples from the PARADIGM 2 and PARADIGM 4 trials; pre-dose (trough) samples for 11 patients (aged 13-65 years old) were collected over the course of 2.7 years starting from trial initiation. Cut-off dates for data analysis are as follows: PARADIGM 2, 19 Mar 2013; PARADIGM 4, 21 Mar 2014; PARADIGM 5, 01 Oct 2018. A ${ }^{1}$ H-NMR-based assay was used to investigate total PEG concentrations in these pre-dose plasma samples, as described below. FIX activity was determined in PARADIGM 2, 4 and 5 using a one-stage activated partial thromboplastin timebased clotting assay (SynthAFax ${ }^{\circledR}$, Instrumentation Laboratory, Holliston, MA, USA) and a product-specific reference standard [9].

\subsection{Analysis of PEG Concentration Using ${ }^{1} \mathrm{H}-\mathrm{NMR}$}

Patient plasma samples $(165 \mu \mathrm{L})$ were mixed with $90 \mu \mathrm{L}$ NMR buffer $(0.56 \mathrm{M}$ sodium phosphate and $0.3 \%$ sodium azide in deuterated water, $\mathrm{pH}$ 7.4) in Eppendorf tubes. Eppendorf tubes were then vortexed and samples transferred to $3 \mathrm{~mm}$ NMR tubes. Samples in NMR tubes were kept refrigerated until analysis. Single samples were analysed using a Bruker Avance IIIHD (Bruker, Germany) instrument equipped with a 5-mm broadband observe (BBO) cryoprobe operating at $400 \mathrm{MHz}$. NMR spectra were recorded at a sample temperature of $27^{\circ} \mathrm{C}$ with a 40 -mm sample filling height. The stebpgp1s191d pulse program (Bruker pulse program library) was applied to acquire diffusion-filtered ${ }^{1} \mathrm{H}$-spectra in Topspin (version 3.5pl6) (Bruker, Germany). Total PEG in plasma (conjugated and free) was determined through the identification of repetitive ethyleneoxy groups comprising PEG chains, which provide a unique and strong resonance signal at a chemical shift of approximately $3.6 \mathrm{ppm}$ that increases with increasing PEG concentration.

The general analytical setup was as follows: an aqueous N9-GP solution (provided by Novo Nordisk) diluted in FIXdeficient haemophilia B plasma served as the reference compound. Standard curve samples had PEG concentrations of $0,0.75,1,3,5,7,9$, and $15 \mu \mathrm{g} / \mathrm{mL}$ and control samples had PEG concentrations of 1,3 , and $9 \mu \mathrm{g} / \mathrm{mL}$. Standards were prepared as a single solution and then aliquoted in replicate or triplicate, depending on the number of samples to be analysed. Standards were analysed 2-6 times and controls analysed randomly between samples in order to assess analytical stability over the measurement period. The lower limit of quantification (LLOQ) for PEG in human plasma was $0.75 \mu \mathrm{g} / \mathrm{mL}$.

\subsection{Data Analysis}

Data analysis was performed and plots generated using R studio (Rstudio Inc, version 1.1.456) and GraphPad Prism (GraphPad Software Inc, version 7.04).

Visual inspection of the individual and mean trough concentrations determined whether steady state had been reached. Steady state was deemed to have been reached when trough concentrations did not change meaningfully between time points, reaching a plateau.

\section{Results}

\subsection{Plasma Levels of 40-kDa PEG}

Steady-state plasma 40-kDa PEG levels were reached approximately 6 months after initiation of prophylactic treatment with once-weekly N9-GP 40 IU/kg in children (PARADIGM 5) and adolescents/adults (PARADIGM 2 and 4) (Fig. 1a). When excluding all measurements taken during the first year of treatment (to ensure steady-state levels had been achieved in all individuals), mean ( \pm standard deviation [SD]) steady-state trough (pre-dose) plasma PEG concentrations over the remaining duration of the study were $5.6 \pm 1.4 \mu \mathrm{g} / \mathrm{mL}$ in the paediatric trial (188 samples) and $5.3 \pm 1.8 \mu \mathrm{g} / \mathrm{mL}$ in the adolescent/adult trial (48 samples). The total range for all individuals was $1.6-8.7 \mu \mathrm{g} / \mathrm{mL}$ in the paediatric trial (except for two outlier measurements, which are described below) and $1.7-9.0 \mu \mathrm{g} / \mathrm{mL}$ in the adolescent/ adult trial. As seen in Fig. 1a, following an initial increase in plasma PEG concentrations up to steady state, there was no continued accumulation and PEG concentrations remained at a steady state for up to 6.5 years of once-weekly treatment with N9-GP.

\subsection{Plasma PEG Levels in a Treatment Outlier}

An elevated plasma PEG concentration $(12.7 \mu \mathrm{g} / \mathrm{mL})$ was observed in a single sample collected from one paediatric PARADIGM 5 patient (age 12 years at baseline) at the 3.5-year time point (Fig. 1b). During the first 3 years of treatment, this patient experienced no bleeding episodes. At the 2.5-year time point, the PEG plasma concentration was measured at $3.29 \mu \mathrm{g} / \mathrm{mL}$, which was within the range observed for the group as a whole. At age 15 years, personal circumstances impacted treatment adherence and the patient reported a series of four bleeds over a 3-month period that were treated with 23 additional doses during four hospitalisations, leading to FIX activity levels $>200 \%$ (Table 1). At the 3.5-year time point, the patient's plasma PEG concentration was measured at $12.7 \mu \mathrm{g} / \mathrm{mL}$. After the patient resumed his normal prophylaxis schedule, the plasma PEG 

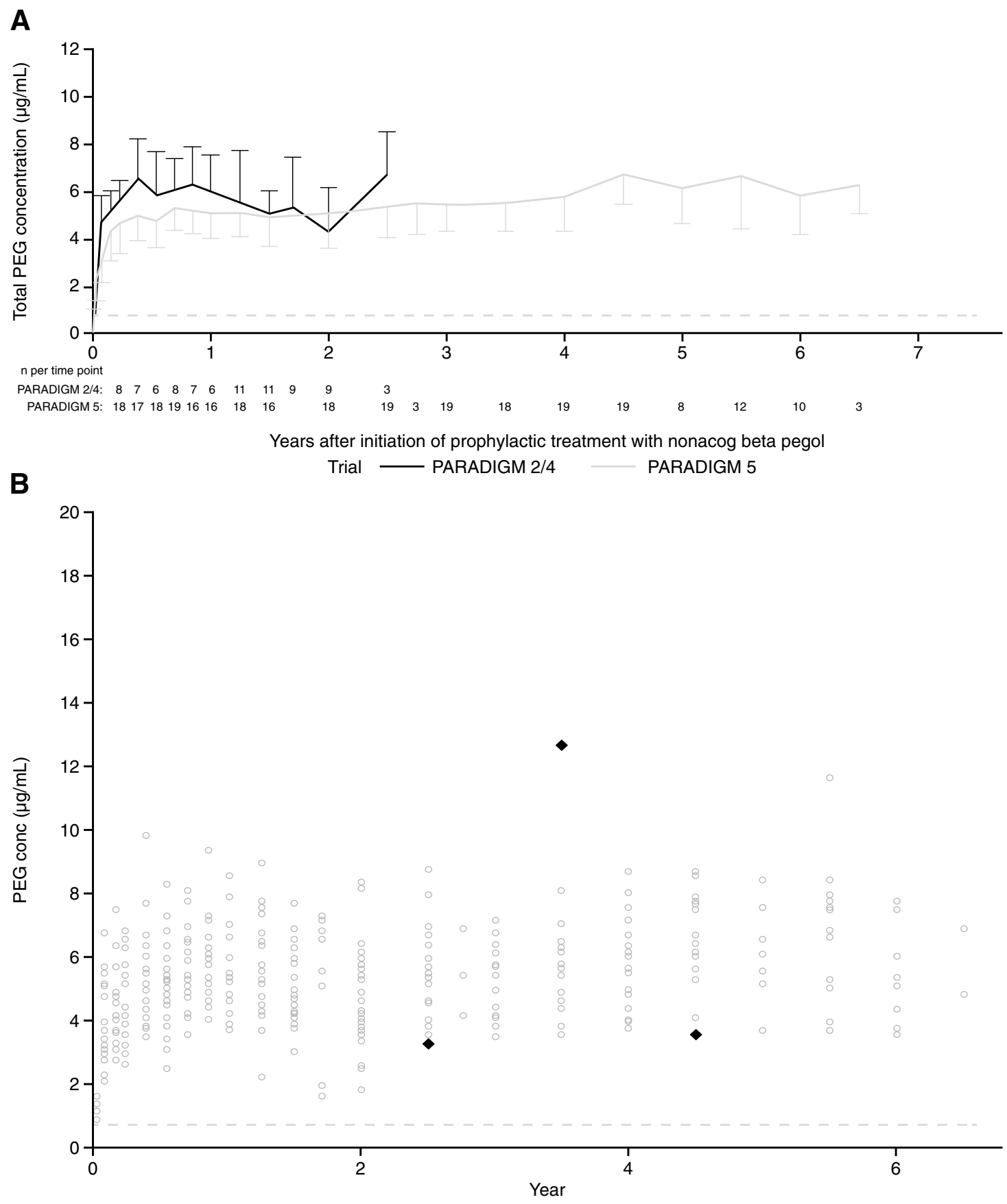

Fig. 1 Pre-dose PEG plasma concentrations in children and adolescents/adults treated with once-weekly $40 \mathrm{IU} / \mathrm{kg}$ N9-GP. a Mean trough plasma PEG concentrations measured in paediatric (PARADIGM 5; grey line) and adolescent/adult patients (PARADIGM 2 and 4 ; black line) over a period of up to 6.5 years of once-weekly prophylactic treatment with N9-GP. Nominal time points are shown. Bars show \pm SD. b One paediatric patient in PARADIGM 5 experienced several bleeding events prior to the 3.5 -year measurement point that were treated with additional N9-GP infusions (see Table 1). This resulted in an increase in plasma PEG concentration. PEG concentration returned to previous levels after the normal prophylactic treatment schedule was resumed. Earlier time points were not analysed for this patient and are not included in a. Individual PARADIGM 5 data points are shown in grey; the affected patient is indicated with black diamonds. The dashed line in panels $\mathbf{a}$ and $\mathbf{b}$ indicates the lower limit of quantification $(0.75 \mu \mathrm{g} / \mathrm{mL})$. N9-GP nonacog beta pegol, $P E G$ polyethylene glycol, $S D$ standard deviation 
Table 1 Treatment regimen for an outlier patient in PARADIGM 5

\begin{tabular}{llll}
\hline Date & Type of bleed & Location & N9-GP dose \\
\hline $09-21$ Oct 15 & Traumatic muscle bleed & Thigh & $\begin{array}{c}1 \times 80 \mathrm{IU} / \mathrm{kg} \\
11 \times 40 \mathrm{IU} / \mathrm{kg} \\
(1-2 \text { per day } \\
\text { over } 8 \text { days })\end{array}$ \\
& & & $3 \times 40 \mathrm{IU} / \mathrm{kg}$ \\
24 Oct 15-04 Nov 15 & & Mouth and nose & $1 \times 40 \mathrm{IU} / \mathrm{kg}$ \\
$10-15$ Nov 15 & Spontaneous allergic rhinitis-induced bleeds & Mouth and nose & $7 \times 40 \mathrm{IU} / \mathrm{kg}$ \\
22 Nov 15-12 Dec 15 & Spontaneous allergic rhinitis-induced bleeds & Right iliopsoas & Spontaneous muscle bleed \\
\hline
\end{tabular}

A PARADIGM 5 patient, aged 15 years, reported a series of four bleeds over a 3-month period that were treated with 23 additional doses during a total of four hospitalisations. Plasma PEG concentrations for this patient are shown in Fig. 1b

N9-GP nonacog beta pegol, PEG polyethylene glycol

concentration had returned to $3.57 \mu \mathrm{g} / \mathrm{mL}$ at the 4.5 -year time point, which was similar to the concentration measured prior to the period of intense N9-GP treatment. This patient did not experience any safety issues associated with this intense treatment period. He is considered a treatment outlier and was later withdrawn from the paediatric trial for other non-safety-related reasons. The three measurements for this patient formed part of an initial exploratory analysis of plasma PEG concentrations in paediatric patients, and informed consent was only obtained for these three samples. Earlier time points were therefore not analysed for this patient and are not included in Fig. 1a.

\subsection{PEG Exposure at Steady State by Age}

To examine whether patient age has an impact on PEG plasma levels following treatment with N9-GP, individual PEG measurements from 19 patients in the paediatric trial and 11 patients in the adolescent/adult trial were split by the age at which the sample was collected into the following groups: $<7,7-12$ and $>12$ years old. Once again, samples collected during the first year of N9-GP exposure were excluded from the analysis to ensure that steady-state levels had been reached for all subjects. Younger patients $(<7$ years old) had lower mean $( \pm$ SD) steady-state PEG plasma concentrations when treated with the same $40 \mathrm{IU} / \mathrm{kg}$ treatment regimen as older children, adolescents and adults: $<7$ years old, $4.6 \pm 0.9 \mu \mathrm{g} / \mathrm{mL}$; $7-12$ years old, $5.9 \pm 1.3 \mu \mathrm{g} / \mathrm{mL}$; $>12$ years old, $5.7 \pm 1.8 \mu \mathrm{g} / \mathrm{mL}$ (Fig. 2 ). Because some patients are represented in more than one group as they grew older during the trials, the number of unique patients in each group is: $<7$ years old, 9 patients; $7-12$ years old, 17 patients; $>12$ years old, 18 patients.

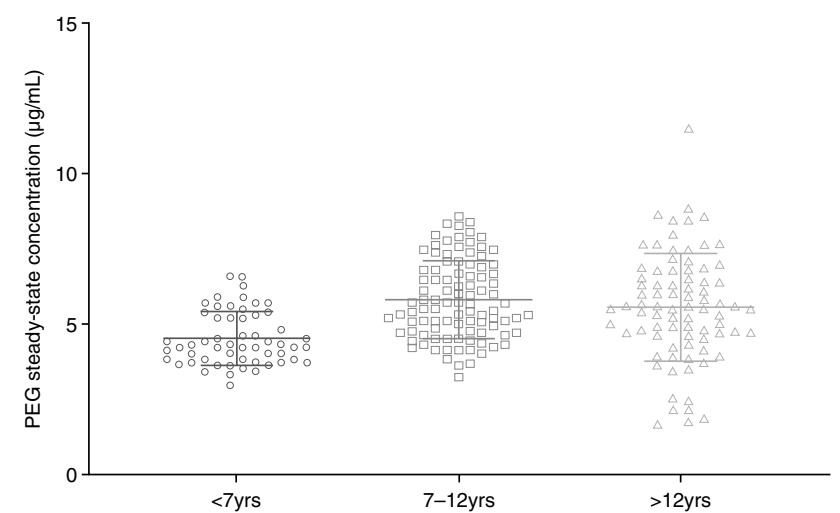

Fig. 2 Pre-dose PEG plasma concentrations by age at time of sampling in children and adolescents/adults treated with N9-GP. Individual PEG measurements split by age at time of sampling are shown for all patients from PARADIGM 2, 4 and 5 that were aged $<7$ years $(n=56), 7-12$ years $(n=97)$ and $>12$ years $(n=82)$. Only measurements taken after at least 12 months of N9-GP treatment were included to ensure PEG levels had reached a steady state for all patients. Bars show mean \pm SD. N9-GP nonacog beta pegol, PEG polyethylene glycol, $S D$ standard deviation

\subsection{Correlation Between Trough Plasma PEG Steady-State Levels and FIX Activity Levels}

A sub-analysis was conducted specifically for the paediatric trial to assess the link between FIX activity levels and 40-kDa PEG plasma concentrations after repeated onceweekly dosing with N9-GP. FIX and PEG levels from the same time point and patient were plotted for each of the 19 paediatric patients. A clear correlation between FIX and PEG levels was observed in plasma (higher FIX levels were associated with higher PEG levels) (Fig. 3a). In addition, mean PEG concentration and FIX activity profiles for 
patients aged 0-6 years and 7-12 years at trial initiation provided further evidence for a relationship between PEG and FIX activity levels (Fig. 3b, c).

In addition to the outlier described above, who exhibited a single elevated plasma PEG measurement, another paediatric patient (aged 10 years at baseline) had a single elevated PEG measurement at 5.5 years of treatment $(11.7 \mu \mathrm{g} / \mathrm{mL})$. This measurement point corresponded to an elevated FIX level of $88.5 \%$ (Fig. 3c). Plasma PEG concentrations observed in this patient before the 5.5 -year time point ranged between 1.6 and $8.7 \mu \mathrm{g} / \mathrm{mL}(N=12$ measurements), which are in line
Fig. 3 Relationship between plasma trough levels of FIX activity and 40-kDa PEG after once-weekly prophylactic dosing of N9-GP in PARADIGM 5 patients. a Data show individual patient measurements of FIX activity and PEG concentration taken at the same time point. The solid line represents a linear regression of the data. b, $\mathbf{c}$ Mean PEG concentration $(\mu \mathrm{g} / \mathrm{mL})$ and FIX activity (IU/mL) profiles for patients $0-6$ years of age (b) and 7-12 years of age (c) at trial initiation receiving weekly prophylactic dosing of N9-GP for up to 6.5 years. Profiles are overlaid to reveal patterns between both measurements. Note that different scales are used for each. Predose FIX measurements were included if they were collected 5-10 days after the last dose and at least 14 days after the last bleeding episode. Bars show \pm SD. FIX factor IX, N9-GP nonacog beta pegol, $P E G$ polyethylene glycol, $S D$ standard deviation
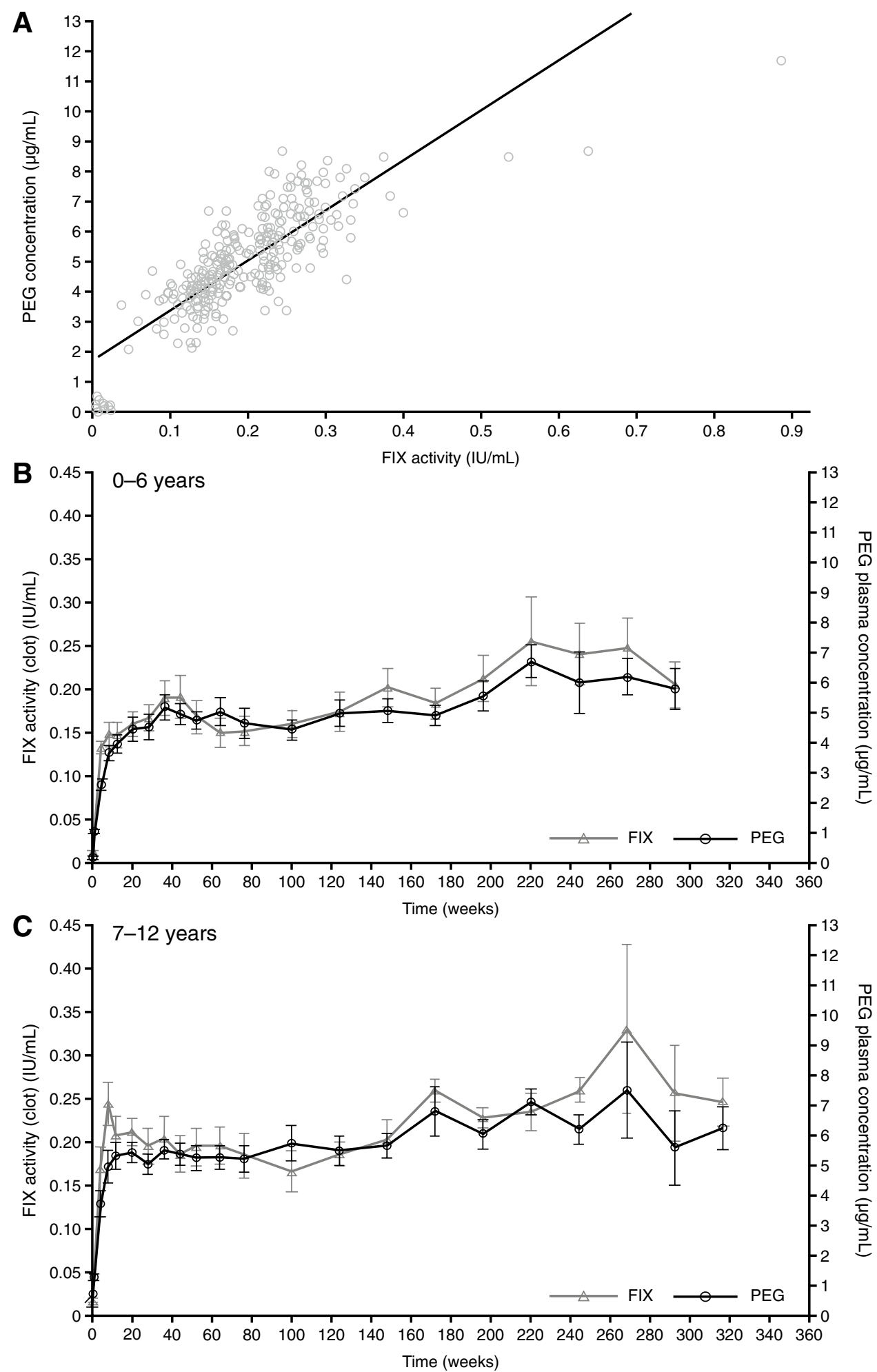
with steady-state plasma PEG concentrations measured in other paediatric patients.

\section{Discussion}

Results presented here show that after approximately 6 months of once-weekly intravenous prophylactic treatment with N9-GP $40 \mathrm{IU} / \mathrm{kg}$, steady-state plasma PEG levels were reached in children, adolescents and adults. Importantly, no unexpected increase was observed over a treatment period lasting for up to 6.5 years.

No overall difference in mean plasma PEG concentrations was observed between the two trials. On first glance, mean PEG levels might be expected to be lower in the paediatric trial due to higher N9-GP clearance rates in children. However, as detailed below, the fact that many of these patients reached adolescence during the trial period of 6.5 years could explain the similarity in mean PEG concentration values between studies.

Although the mean steady-state plasma PEG level in paediatric patients appears to increase slightly over time after reaching initial steady-state levels, it is clear from individual PEG and FIX activity data that this is not related to unexpected PEG accumulation. Instead, this can be attributed to concurrently rising FIX activity levels in approximately half of the children in the trial (the other children showed flat PEG and FIX levels throughout) (individual data not shown). A clear correlation between plasma FIX and PEG levels was observed (Fig. 3).

Younger patients exhibited lower plasma PEG levels compared with older children, adolescents and adults. As mentioned above, the paediatric trial included children aged $\leq 12$ years old at trial initiation, meaning that many participants became adolescents over the course of the study. Therefore, the slight increase in PEG over the 6.5-year duration of the trial can be explained by a gradual increase in FIX levels due to physiological changes and decreased FIX clearance (per kg body weight) as the children grew older. This is substantiated by the age-dependent increases in mean steady-state FIX trough activity levels reported previously (children $0-6$ years: $15 \%$; children $7-12$ years: 19\%; adolescents/adults: 27\%) [3,7]. The clearance effect for younger patients is therefore diminished when looking at PARADIGM 5 patients as a whole because the children grew older during the study. Moreover, sporadically elevated FIX activity levels due to treatment of bleeds or surgical procedures may be associated with a temporary and parallel elevation in PEG plasma levels, as has been observed in the outlier cases described here.

With regard to PEG exposure, the treatment outlier described in Fig. 1b provides two important pieces of information. Firstly, even after receiving a rather intense dosing regimen with N9-GP (on top of prophylaxis), plasma PEG trough levels did not appear to increase drastically. Secondly, plasma PEG levels returned to the previously observed steady-state level after resumption of normal prophylactic N9-GP treatment, confirming that PEG is cleared from plasma upon treatment cessation or reduction and that PEG kinetics are predictable.

Although it is not feasible to measure cell/tissue PEG concentrations in patients using currently available analysis methods, single-dose distribution and excretion studies with N9-GP-treated rats showed that 40-kDa PEG was widely distributed [17]. While elimination rates varied, intact 40-kDa PEG was excreted over time from all tissues and excreted in urine and faeces [17]. The observed terminal half-lives in rat plasma and tissues ranged from 15 to 49 days, indicating that a steady state will be reached in all human tissues within 1-2 years of exposure (based on allometric scaling) [20].

The potential effect on safety of total 40-kDa PEG levels from N9-GP dosing during steady state was investigated in a chronic toxicity study in rats (dose levels up to $1200 \mathrm{IU} / \mathrm{kg}$ delivered every 5th day). Although no PEG measurements were performed in the study, steady-state 40-kDa PEG concentrations $\sim$ sixfold above clinical exposure levels were predicted (from single-dose rat data) in all tissues [21]. No adverse reaction was observed in the animals and there were no treatment-related histopathological changes in any tissue.

With regard to the clinical safety profile of N9-GP, the PARADIGM trials include exposure in children $<12$ years of age for up to 6.5 years and up to 2.7 years' exposure in adolescents and adults $\geq 12$ years of age $[2,7]$. Analyses from the PARADIGM trials showed that the safety profile of N9-GP to date is similar to that of other non-PEGylated FIX products and did not identify any safety issues that could be related to PEG.

In this context, a recent study from Stidl et al. examined trends in adverse events reported in paediatric populations treated with a number of different PEGylated drugs, including N9-GP, as well as coagulation factor VIII and FIX replacement therapies that include PEG as an excipient [22]. No consistent pattern of adverse events potentially attributable to PEG exposure emerged for any of the PEGylated products.

Further, a number of other PEGylated products are available on the market that contain a $40-\mathrm{kDa}$ PEG moiety similar to that used in N9-GP, including certolizumab pegol $\left(\right.$ Cimzia $\left.^{\circledR}\right)$, pegylated interferon alfa-2a (Pegasys ${ }^{\circledR}$ ) and pegaptanib sodium injection (Macugen ${ }^{\circledR}$ ) [16]. The most relevant comparator to N9-GP, based on dosing regimen and indication for long-term use, is certolizumab pegol, which has been approved since 2008 for Crohn's disease and other inflammatory indications, including in pregnant women [16]. A Cochrane review showed that certolizumab pegol did not significantly differ from other non-PEGylated 
anti-TNF agents in the rate of total adverse events or the rate of withdrawals due to adverse events following use [23, 24].

To put the total 40-kDa steady-state plasma PEG exposure after N9-GP dosing into clinical perspective, recent studies from Mahadevan et al. and Mariette et al. evaluated plasma 40-kDa PEG concentrations in pregnant women ( $>18$ years old) dosed prophylactically with certolizumab pegol (200 mg every 2 weeks or $400 \mathrm{mg}$ every 4 weeks) $[18,19]$. For maternal plasma samples taken on the day of delivery, Mariette et al. reported median PEG concentrations of approximately $30 \mu \mathrm{g} / \mathrm{mL}$ (range 10-60 $\mu \mathrm{g} / \mathrm{mL}$; $N=16$ ), and Mahadevan et al. reported mean PEG concentrations of $34 \mu \mathrm{g} / \mathrm{mL}$ (range $11-75 \mu \mathrm{g} / \mathrm{mL} ; N=10$ ) [18, 19]. These plasma levels are approximately fivefold higher than the mean steady-state PEG plasma concentrations measured in the PARADIGM trials. Thus, N9-GP treatment, as reported here, results in lower PEG steady-state levels than that observed with certolizumab pegol. Although the biodistribution of two PEG-conjugated molecules can differ due to the protein moiety [22], a significant fraction of the circulating PEG would be expected to be present as free PEG at steady state, with distribution and elimination of the free PEG expected to be similar. Thus, a comparison between two different 40-kDa PEG conjugates is considered relevant.

Taken together, there are no direct or indirect indications that steady-state exposure to 40-kDa PEG after prophylactic, once-weekly, N9-GP dosing causes any safety concerns or unexpected PEG-related adverse events.

\section{Conclusion}

The data presented here show that $40-\mathrm{kDa}$ PEG levels reach steady state in plasma within 6 months following initiation of once-weekly prophylactic intravenous treatment with N9-GP in children, adolescents and adults. This indicates that PEG is eliminated over time and does not continue to accumulate during repeated administration of N9-GP. Further, a clear correlation exists between plasma FIX activity and PEG levels.

Acknowledgements The authors would like to thank Rikke Zillmer and Kristine Beisner for their assistance with data collection, Kerstin Pietzko for support in generating figures, and Bent O. Petersen for help with sample analysis. Medical writing support was provided by Physicians World Europe GmbH, Mannheim, Germany, supported by Novo Nordisk.

\section{Compliance with Ethical Standards}

Funding This study was funded by Novo Nordisk A/S.
Conflict of interest Ola Sternebring, Charlotte Gabel-Jensen, Helene Jacobsen, Andrew James Benie and Inga Bjørnsdottir are employees of Novo Nordisk A/S and are Novo Nordisk A/S shareholders.

Ethical approval All procedures performed in studies involving human participants were in accordance with the ethical standards of the institutional and/or national research committee and with the 1964 Helsinki declaration and its later amendments or comparable ethical standards.

Informed consent Informed consent was obtained from all individual participants included in the study or their legally appointed representatives.

Open Access This article is distributed under the terms of the Creative Commons Attribution-NonCommercial 4.0 International License (http://creativecommons.org/licenses/by-nc/4.0/), which permits any noncommercial use, distribution, and reproduction in any medium, provided you give appropriate credit to the original author(s) and the source, provide a link to the Creative Commons license, and indicate if changes were made.

\section{References}

1. Srivastava A, Brewer AK, Mauser-Bunschoten EP, Key NS, Kitchen S, Llinas A, et al. Guidelines for the management of hemophilia. Haemophilia. 2013;19(1):e1-47. https://doi.org/10. 1111/j.1365-2516.2012.02909.x.

2. Collins PW, Young G, Knobe K, Karim FA, Angchaisuksiri P, Banner $\mathrm{C}$, et al. Recombinant long-acting glycoPEGylated factor IX in hemophilia $\mathrm{B}$ : a multinational randomized phase 3 trial. Blood. 2014;124(26):3880-6. https://doi.org/10.1182/blood -2014-05-573055.

3. Tiede A, Abdul-Karim F, Carcao M, Persson P, Clausen WHO, Kearney S, et al. Pharmacokinetics of a novel extended half-life glycoPEGylated factor IX, nonacog beta pegol (N9-GP) in previously treated patients with haemophilia B: results from two phase 3 clinical trials. Haemophilia. 2017;23(4):547-55. https://doi. org/10.1111/hae.13191.

4. Negrier C, Knobe K, Tiede A, Giangrande P, Moss J. Enhanced pharmacokinetic properties of a glycoPEGylated recombinant factor IX: a first human dose trial in patients with hemophilia B. Blood. 2011;118(10):2695-701. https://doi.org/10.1182/blood -2011-02-335596.

5. Ostergaard H, Bjelke JR, Hansen L, Petersen LC, Pedersen AA, Elm T, et al. Prolonged half-life and preserved enzymatic properties of factor IX selectively PEGylated on native $N$-glycans in the activation peptide. Blood. 2011;118(8):2333-41. https://doi. org/10.1182/blood-2011-02-336172.

6. Jimenez-Yuste V, Auerswald G, Benson G, Lambert T, Morfini $\mathrm{M}$, Remor E, et al. Achieving and maintaining an optimal trough level for prophylaxis in haemophilia: the past, the present and the future. Blood Transfus. 2014;12(3):314-9. https://doi. org/10.2450/2014.0298-13.

7. Carcao M, Zak M, Abdul Karim F, Hanabusa H, Kearney S, Lu MY, et al. Nonacog beta pegol in previously treated children with hemophilia B: results from an international open-label phase 3 trial. J Thromb Haemost. 2016;14(8):1521-9. https://doi. org/10.1111/jth. 13360 .

8. Escobar MA, Tehranchi R, Karim FA, Caliskan U, Chowdary P, Colberg T, et al. Low-factor consumption for major surgery in haemophilia B with long-acting recombinant glycoPEGylated factor 
IX. Haemophilia. 2017;23(1):67-76. https://doi.org/10.1111/ hae.13041.

9. Young G, Collins PW, Colberg T, Chuansumrit A, Hanabusa H, Lentz SR, et al. Nonacog beta pegol (N9-GP) in haemophilia B: a multinational phase III safety and efficacy extension trial (paradigm4). Thromb Res. 2016;141:69-76. https://doi.org/10.1016/j. thromres.2016.02.030.

10. van Witteloostuijn SB, Pedersen SL, Jensen KJ. Half-life extension of biopharmaceuticals using chemical methods: alternatives to PEGylation. ChemMedChem. 2016;11(22):2474-95. https:// doi.org/10.1002/cmdc. 201600374 .

11. EMA. Assessment report for Refixia 2017. https://www.ema.europ a.eu/documents/assessment-report/refixia-epar-public-assessment -report_en.pdf. Accessed 16 Oct 2018.

12. FDA. Package insert-ADYNOVATE. 2016. https://www.fda. gov/downloads/BiologicsBloodVaccines/BloodBloodProducts/ ApprovedProducts/LicensedProductsBLAs/FractionatedPlasmaPr oducts/UCM472594.pdf. Accessed 16 Oct 2018.

13. FDA. Package insert-JIVI. 2018. https://www.fda.gov/downl oads/BiologicsBloodVaccines/UCM618979.pdf. Accessed 16 Oct 2018.

14. Turecek PL, Bossard MJ, Schoetens F, Ivens IA. PEGylation of biopharmaceuticals: a review of chemistry and nonclinical safety information of approved drugs. J Pharm Sci. 2016;105(2):460-75. https://doi.org/10.1016/j.xphs.2015.11.015.

15. Baumann A, Tuerck D, Prabhu S, Dickmann L, Sims J. Pharmacokinetics, metabolism and distribution of PEGs and PEGylated proteins: quo vadis? Drug Discov Today. 2014;19(10):1623-31. https://doi.org/10.1016/j.drudis.2014.06.002.

16. Ivens IA, Achanzar W, Baumann A, Brandli-Baiocco A, Cavagnaro J, Dempster M, et al. PEGylated biopharmaceuticals: current experience and considerations for nonclinical development. Toxicol Pathol. 2015;43(7):959-83. https://doi.org/10.1177/01926 23315591171.

17. Sternebring O, Christensen JK, Bjornsdottir I. Pharmacokinetics, tissue distribution, excretion, and metabolite profiling of
PEGylated rFIX (nonacog beta pegol, N9-GP) in rats. Eur J Pharm Sci. 2016;92:163-72. https://doi.org/10.1016/j.ejps.2016.06.025.

18. Mahadevan U, Wolf DC, Dubinsky M, Cortot A, Lee SD, Siegel CA, et al. Placental transfer of anti-tumor necrosis factor agents in pregnant patients with inflammatory bowel disease. Clin Gastroenterol Hepatol. 2013;11(3):286-92; quiz e24. https://doi. org/10.1016/j.cgh.2012.11.011.

19. Mariette X, Forger F, Abraham B, Flynn AD, Molto A, Flipo $\mathrm{RM}$, et al. Lack of placental transfer of certolizumab pegol during pregnancy: results from CRIB, a prospective, postmarketing, pharmacokinetic study. Ann Rheum Dis. 2018;77(2):228-33. https:// doi.org/10.1136/annrheumdis-2017-212196.

20. EMA. Refixia: EPAR product information. 2017. https://www. ema.europa.eu/documents/product-information/refixia-epar-produ ct-information_en.pdf. Accessed 19 Oct 2018.

21. Rasmussen CE, Nowak J, Larsen JM, Bottomley A, Rowles A, Offenberg $\mathrm{H}$. Evaluation of nonacog beta pegol long-term safety in the immune-deficient Rowett nude rat (Crl:NIH-Foxn1rnu). Toxicol Pathol. 2016;44(5):726-37. https://doi.org/10.1177/01926 23316633311.

22. Stidl R, Denne M, Goldstine J, Kadish B, Korakas KI, Turecek PL. Polyethylene glycol exposure with antihemophilic factor (recombinant), PEGylated (rurioctocog alfa pegol) and other rherapies indicated for the pediatric population: history and safety. Pharmaceuticals (Basel). 2018. https://doi.org/10.3390/ph11030075.

23. Mease PJ. Certolizumab pegol in the treatment of rheumatoid arthritis: a comprehensive review of its clinical efficacy and safety. Rheumatology (Oxford). 2011;50(2):261-70. https://doi. org/10.1093/rheumatology/keq285.

24. Singh JA, Wells GA, Christensen R, Tanjong Ghogomu E, Maxwell L, Macdonald JK, et al. Adverse effects of biologics: a network meta-analysis and Cochrane overview. Cochrane Database Syst Rev. 2011;2:CD008794. https://doi.org/10.1002/14651858. cd008794.pub2. 